\title{
Editorial
}

\section{Time for a change}

Ten years have passed since I agreed to help Blackwell Science launch a new journal covering all aspects of patient and public participation in health care and health policy. You could find research papers on this topic at that time, but they were widely dispersed across a large number of journals and there was no central focus for creative development and critical debate. We felt a specialist journal was needed to fill the gap.

The first issue of Health Expectations appeared in June 1998. This set the international tone with research papers on the use of evidence by Norwegian patient organisations, papers from Canada and the USA on patient decision aids for breast cancer and coronary angiography, and one from the Netherlands on what patients can do to improve health care. Julian Tudor Hart kicked it off with a stimulating article on the development of health systems in the US and UK. He called for revolutionary change in the way care is conceived, organized and resourced by making professionals accountable to patients as their intelligent partners. ${ }^{1}$

Since then we have published 36 issues containing more than 250 papers from around the world on a wide variety of topics. The theory and practice of shared decision-making and the use and effects of patient decision aids has been a very strong theme, but other issues such as lay involvement in service development and priority-setting, the activities of patient organisations, information and communications, and patient participation in research have attracted a large number of high quality submissions.

We have learnt a great deal since the journal was launched. We now have a better understanding of patients' information preferences and of the circumstances in which they want to be involved in decisions about their care. We know that decision aids can improve patients' knowledge and understanding of their condition and of the treatment options and outcome probabilities; that educational programmes for patients with chronic conditions can improve health outcomes; and that patient-held records can enhance their sense of empowerment. We have shared information about the potential for patient organisations and community groups to get engaged in determining local priorities and in shaping service developments; and we have a deeper understanding of ways in which involving service users in the research process can improve the relevance and utility of health services research.

The task is not over, however. There are still many gaps in our collective knowledge and many opportunities for innovative research in this important field. For example, we need more and better evaluations of strategies for building health literacy, especially those that can shed light on the factors that mediate and moderate the relationship between health literacy and health outcomes. We need to establish the most effective ways of delivering health information and education, paying particular attention to communication with low literacy, hard-to-reach and disadvantaged groups.

We must pay greater attention to the problems of translating research evidence on shared decision-making into practical strategies for implementation in the real world of mainstream clinical practice. This should involve more demonstration projects to test the feasibility and effects of providing decision support to patients in a wide range of settings, and more studies looking at how best to communicate risk and probability to lay audiences. 
How best to manage chronic disease is a priority question for almost all health systems and Health Expectations could make an important contribution to the policy debate by publishing research that addresses the main knowledge gaps. For example, little is known about the long term outcomes of strategies to support selfmanagement and the comparative effectiveness, including cost-effectiveness, of different approaches to supporting patients' self-care efforts. It seems clear that there are no magic bullets to be discovered, but we need more fine-grained studies to address the question of which components of complex interventions provide the greatest benefit.

The part that patients can play in improving the safety of their care has been flagged up as an important priority, but has largely failed to attract the attention of researchers. Health Expectations has tried to fill the gap and an article in this issue reviews some relevant research. ${ }^{2}$ We need to investigate patients' willingness and ability to be involved in the safety of their care and to identify the issues and settings where patient involvement is most achievable and will have the greatest impact. To what extent and in what ways do patients want to take a role in safety improvement?

Access to care and health advice and how to overcome the barriers to equitable access is another important topic for those concerned about the patient's perspective. Certain groups have long faced difficulties in accessing appropriate, high quality health care, and traditional access routes are no longer sufficient to meet the diversity of patients' needs. New technologies, such as electronic communications and the internet, offer tremendous scope for tackling previously intractable access problems, but we need a better understanding of how to use these to best effect and how to minimise any adverse impact.

You might imagine that strategies for improving patients' experience of health care would have attracted a great deal of research, but the weakness of the evidence base is striking. There is a great deal we don't know about how to address the problems identified in numerous patient surveys. For example, why do younger patients, those with lower incomes or poorer health, and those from minority ethnic groups report worse experiences than those in other groups? What is the best way to encourage providers to improve service quality? What are the effects of offering patients a choice of provider? What are the positive and negative effects of public disclosure of performance information? What are the benefits and risks of encouraging patient advocates to get involved? What is the best way to organise a complaints system? How can we most effectively involve patients and lay people in planning and evaluating service developments?

These questions should provide plenty of meat for Health Expectations contributors, reviewers and readers to get their teeth into. I have confidence that these issues can be resolved and that journals such as this have a great contribution to make, but after ten years at the journal's helm I have decided it's time to step down and give someone else a turn.

I am delighted to announce that Vikki Entwistle, Professor of Values in Health Care at the Social Dimensions of Health Institute, in Scotland's Universities of Dundee and St Andrews, has agreed to take over the editorship of Health Expectations. Vikki has been deputy editor of the journal since its inception, so she knows it inside out. I am confident that I'm leaving the journal in very capable hands and that its importance and impact (the current impact factor is 2.089) will continue to grow from strength to even greater strength.

\section{Angela Coulter \\ Editor}

\section{References}

1 Hart JT. Expectations of health care: promoted, managed or shared? Health Expect, 1998; 1: 3-13.

2 Davis RE, Jacklin R, Sevdalis N, Vincent CA. Patient involvement in patient safety: what factors influence patient participation and engagement. Health Expect, 2007; 10: 259-267. 